Pacific Journal of Mathematic 


\title{
TRAJECTORY INTEGRALS OF SET VALUED FUNCTIONS
}

\author{
T. F. BRIDGLAND, JR.
}

Let $I$ be a compact interval of the real line and for each $t$ in $I$, let $F(t)$ denote a nonvoid subset of euclidean $n$-space $E^{n}$. Let $\mathscr{F}_{I}(F)$ be the collection of all Lebesgue summable functions $u: I \rightarrow E^{n}$ having the property that $u(t) \in F(t)$ almost everywhere on $I$. Following the lead of Kudo and Richter, Aumann defines the integral of $F$ over $I$ by

$$
\int_{I} F(t) d t=\left\{\int_{I} f(t) d t \mid f \in \mathscr{F}_{I}(F)\right\}
$$

and, in addition to other results, establishes a dominated convergence theorem for such integrals. Hermes has pursued Aumann's line of thought to obtain results concerning something akin to a "derivative" for set valued functions.

It is certainly also valid (and for control theoretic applications essential) to define the trajectory integral of $F$ to be the set $\mathscr{S}_{I}(F)$ of all functions which vanish at the left endpoint of $I$ and have derivatives in $\mathscr{F}_{I}(F)$. The purpose of this paper is taken to be the study of the trajectory integrals of nonvoid, compact set valued functions. A primary goal is the extension of the results of Aumann to include the trajectory integral. A secondary goal is the provision of an intuitively meaningful definition of "derivative" for set valued functions.

Whereas $\int_{I} F(t) d t$ is a subset of $E^{n}, \mathscr{S}_{I}(F)$ is a subset of a space of functions on $I$ to $E^{n}$. Taking note of the relation

$$
\int_{[0, t]} F(\tau) d \tau=\left\{\mu(t) \mid \mu \in \mathscr{S}_{I}(F)\right\}, t \in I,
$$

the validity of which is obvious when $\mathscr{F}_{I}(F)$ is nonvoid, it is clear that the distinction between $\mathscr{S}_{I}(F)$ and $\int_{[0, t]} F(\tau) d \tau$ is essentially that between "function" and "value of a function". In view of this distinction, one necessarily anticipates that a study of the trajectory integral would, in some sense, subsume that of the integral defined by Aumann. ${ }^{1}$ Concrete justification for this point of view already exists in control theory [4].

Further motivation for the study of the trajectory integral arises in connection with the existence theory of the generalized differential equation

1 The work of Kudo, Richter, Aumann and Hermes cited previously is to be found in references $[\mathbf{1 3}],[\mathbf{1 8}],[\mathbf{1}]$ and $[\mathbf{1 1}]$ respectively. 
in the case in which the set valued function satisfies, in particular, a condition of measurability in its first argument. Here one anticipates that a suitably formulated dominated convergence theorem for the trajectory integral would provide the means for a constructive proof of existence, along classical lines, thereby providing at same time a method of approximation to solutions. This is a question of no little importance inasmuch as the general existence theorem of Pliś [17] and Castaing [5] has been established by nonconstructive methods.

The goals of this paper are achieved in the following way. After developing, in $\S 1$, the pertinent algebraic and topological properties of the space $\Omega^{n}$ of nonvoid compact subsets of $E^{n}$, in $\S 2$ we establish several fundamental structural properties of Lebesgue measurable functions on $E^{1}$ to $\Omega^{n}$. The concept of Lebesgue measurability for functions on $E^{1}$ to $\Omega^{n}$ is due to Plis [16] and is a natural generalization of the concept of measurability of functions with range in $E^{n}$. As Hermes has pointed out [11], Aumann's "Borel measurability" implies measurability in the sense defined by Pliś. Some of the theorems of $\S 2$ have already been stated, without proof and in a somewhat less general form, by Filippov [9]. The central result of $\S 2$ is Theorem 2.3 which is the counterpart of the theorem for point valued functions which asserts that almost every point in the domain of a summable function is a Lebesgue point of the function. This theorem plays an essential role in the proofs of two of the major results of the paper: Theorems 3.1 and 5.1.

Theorems 3.1 and 3.2 are the principal results of interest in $\S 3$. In the former, conditions are stated-the chief one of which is measurability of $F$-under which $\mathscr{S}_{I}(F)$ is a nonvoid compact subset of each of two linear topological (function) spaces. One of these compactness properties, together with Hermes' refinement [12, Lemma 1.2] of Filippov's "measurable selection" lemma [8], permits a short proof of the dominated convergence theorem (Theorem 3.2) in a form suited to the proof of the existence theorem (Theorem 4.1) for (2). In $\S 3$ we also devote some attention to the relationship between Aumann's results and our own.

Finally, in $\S 5$, we define a derivative for an element of a certain function space which, owing to its obvious relationship to Huygen's principle of wave propagation, we have styled "the Huygens derivative". The principal result (Theorem 5.1) of this section asserts, loosely speaking, that the Huygens derivative of the trajectory integral of a measurable function $F$ is almost everywhere the convex hull of $F(t)$. As easy corollaries to this theorem we obtain generalizations of some of the results of Hermes [11] mentioned previously. 
1. Algebraic and topological preliminaries. In this paper we shall need the following Banach spaces.

$E^{n}$ : euclidean $n$-space, with the scalar product of $a, b \in E^{n}$ denoted by $a \circ b$ and with norm denoted by $\|x\| \equiv$ $(x \circ x)^{1 / 2}$;

$\mathscr{C}^{n}(I): \quad$ space of continuous functions on $I$ to $E^{n}$, with supremum norm $\langle x\rangle=\max \{\|x(t)\| \mid t \in I\}$;

$\mathscr{N} \mathscr{A} \mathscr{C}^{n}(I)$ : space of absolutely continuous functions on $I$ to $E^{n}$, vanishing at the left endpoint of $I$, with norm $\hat{x}=$ $\int_{I}\|\dot{x}(t)\| d t$

$\mathscr{L}_{1}^{n}(I): \quad$ space of Lebesgue summable functions on $I$ to $E^{n}$, with norm $\langle\langle x\rangle\rangle=\int_{I}\|x(t)\| d t$.

In each instance, $I$ denotes a nondegenerate compact interval of $E^{1}$. Throughout this paper the symbol $\phi$ will be used to denote the null set. We shall also need the following classes of subsets of $E^{n}$ and $\mathscr{C}^{n}(I)$ :

$\Omega^{n}: \quad$ class of nonvoid, compact subsets of $E^{n}$;

$\Gamma^{n}$ : $\quad$ class of nonvoid, compact, convex subsets of $E^{n}$;

$\mathscr{Z}^{n}(I)$ : class of nonvoid, compact subsets of $\mathscr{C}^{n}(I)$;

$\mathscr{K}^{n}(I)$ : class of nonvoid, compact, convex subsets of $\mathscr{C}^{n}(I)$.

Definition 1.1. Given a field, $\Phi$, of scalars and a set, $K$, of vectors, together with functions $+: K \times K \rightarrow K$ and $\times: \Phi \times K \rightarrow K$, $K$ is called a quasilinear space over $\Phi$ if and only if all the axioms for a linear space obtain except (i) the distributivity of $\times$ over scalar addition and (ii) the existence of an inverse under + .

Definition 1.2. For $\alpha \in E^{1}, A, B \in \Omega^{n}$,

$$
\begin{aligned}
A+B & =\{a+b \mid a \in A ; b \in B\}, \\
\alpha A & =\{\alpha a \mid a \in A\} .
\end{aligned}
$$

The following result is easy to verify.

LEMma 1.1. With the foregoing definition (Definition 1.2) of addition and scalar multiplication, $\Omega^{n}$ and $\Gamma^{n}$ are quasilinear spaces over the real field.

Definition 1.3. Let $A, B \in \Omega^{n}, Y, Z \in \mathscr{C}^{n}(I)$ and $x \in E^{n}, y \in \mathscr{C}^{n}(I)$; then we may define: 


$$
\begin{gathered}
\alpha(x, A)=\min \{\|x-a\| \mid a \in A\} \\
\beta(y, Z)=\min \{\langle y-z\rangle \mid z \in Z\} \\
\bar{\rho}(B, A)=\max \{\alpha(x, A) \mid x \in B\} \\
\bar{\sigma}(Y, Z)=\max \{\beta(y, Z) \mid y \in Y\} \\
\rho(A, B)=\max \{\bar{\rho}(A, B), \bar{\rho}(B, A)\} \\
\sigma(Y, Z)=\max \{\bar{\sigma}(Y, Z), \bar{\sigma}(Z, Y)\} \\
\nu(A, p)=\max \{p \circ \sigma \mid \sigma \in A\} \quad\|A\|=\rho(A,\{0\}) \\
\bar{\Delta}(A, B)=\max \{\nu(A, p)-\nu(B, p) \mid\|p\|=1\} \\
\quad A_{\eta}=\left\{x \in E^{n} \mid \alpha(x, A) \leqq \eta\right\} \\
\Delta(A, B)=\max \{\bar{J}(A, B), \bar{\Delta}(B, A)\} \\
\quad S(x, p)=\left\{\xi \in E^{n}\|\xi-x\| \leqq p\right\}, p \geqq 0 .
\end{gathered}
$$

Lemma 1.2. (i ) $\left\{\Omega^{n}, \rho\right\},\left\{\Gamma^{n}, \rho\right\},\left\{\mathscr{H}^{n}(I), \sigma\right\}$ and $\left\{\mathscr{K}^{n}(I), \sigma\right\}$ are metric spaces.

(ii) If $A \in \Omega^{n}\left(\in \Gamma^{n}\right)$ then $A_{\eta} \in \Omega^{n}\left(\in \Gamma^{n}\right)$ for all $\eta>0$ and $A_{\eta}=$ $A+S(0, \eta)$

(iii) If $A, B \in \Gamma^{n}$ then $\bar{\rho}(A, B)=\bar{\Delta}(A, B)$ and

$$
\Delta(A, B)=\max \{|\nu(A, p)-\nu(B, p)| \mid\|p\|=1\} .
$$

(iv) If $A, B, C \in \Gamma^{n}$ then $\bar{\rho}(A+B, A+C)=\bar{\rho}(B, C)$.

Proof. The proofs of (i), (ii) and (iii) are to be found in [4]. For (iv), we have, by virtue of (iii),

$$
\begin{aligned}
\bar{\rho}(A+B, A+C) & =\max \{\nu(A+B, p)-\nu(A+C, p) \mid\|p\|=1\} \\
& =\max \{\nu(A, p)+\nu(B, p)-\nu(A, p)-\nu(C, p) \mid\|p\|=1\} \\
& =\bar{\rho}(B, C) .
\end{aligned}
$$

Henceforth we shall use $\Omega^{n}, \Gamma^{n}, \mathscr{C}^{n}(I), \mathscr{C}^{n}(I)$ to denote the metric spaces obtained by virtue of Definition 1.3 and Lemma 1.2 (i) and in the cases of $\Omega^{n}, \Gamma^{n}$ we shall suppose that the algebraic structure of Definition 1.2 has been imposed. For a point $A \in \Omega^{n}$ we shall denote by $A^{*}$ the convex hull of $A$; it is well known that $A^{*} \in \Gamma^{n}$. Moreover, if $\eta \in E^{1}$ and $A, B \in \Omega^{n}\left(\in \Gamma^{n}\right)$ then $\eta A$ and $A+B$ are in $\Omega^{n}$ (in $\left.\Gamma^{n}\right)[6, \mathrm{~V} .1 .4]$.

Lemma 1.3. (i ) If $\eta \in E^{1}$ and $A, B \in \Omega^{n}$ then $\bar{\rho}(\eta A, \eta B)=$ $|\eta| \bar{\rho}(A, B)$.

(ii) If $A, B, C \in Q^{n}$ then $\bar{\rho}\left(B^{*}, C^{*}\right) \leqq \bar{\rho}(A+B, A+C) \leqq \bar{\rho}(B, C)$. (iii) If $A, B, C, D \in \Omega^{n}$ then $\bar{\rho}(A+B, C+D) \leqq \bar{\rho}(A, C)+\bar{\rho}(B, D)$.

Proof. The proof of (i) is trivial. Part (iii) is an easy con- 
sequence of (ii) and the "relaxed" triangle law [4, Lemma 1.1]. The second inequality of (ii) follows readily from the definitions and only the first inequality remains to be proved. By [6, V. 2.4]

$$
\bar{\rho}\left(A^{*}+B^{*}, A^{*}+C^{*}\right)=\bar{\rho}\left((A+B)^{*},(A+C)^{*}\right)
$$

and then by Lemma 1.2 (iv)

$$
\bar{\rho}\left(B^{*}, C^{*}\right)=\bar{\rho}\left((A+B)^{*},(A+C)^{*}\right) .
$$

Now for $D, E \in \Omega^{n}$ we have $D \subset E+S(0, \gamma)$, where $\gamma=\bar{\rho}(D, E)$; hence $D^{*} \subset E^{*}+S(0, \gamma)$ or $D^{*} \subset\left(E^{*}\right)_{\gamma}$ by Lemma 1.2 (ii) from which we conclude $\bar{\rho}\left(D^{*}, E^{*}\right) \leqq \bar{\rho}(D, E)$. Setting $D=A+B, E=A+C$, the first inequality of (ii) follows from this result and the last formula line.

Corollary 1.1. Let $\eta, \gamma \in E^{1}, A, B \in \Omega^{n}$; then

(i) $\|\eta A\|=|\eta|\|A\|$;

(ii) $\|A\| \geqq 0$ and $\|A\|=0$ if and only if $A=\{0\}$;

(iii) $\|A+B\| \leqq\|A\|+\|B\|$;

(iv) $|\|A\|-\|B\|| \leqq \rho(A, B) \leqq\|A\|+\|B\|$;

(v) $\bar{\rho}(\eta A, \gamma A) \leqq|\eta-\gamma|\|A\|$.

Proof. (i) through (iv) follow easily from the definitions and Lemma 1.3. For (v) we have from Lemma 1.3 (i), (ii)

$$
\begin{aligned}
\bar{\rho}(\eta A, \gamma A)= & |\eta-\gamma| \bar{\rho}\left(\left(1+\frac{\gamma}{\eta-\gamma}\right) A,\left(\frac{\gamma}{\eta-\gamma}\right) A\right) \\
& \leqq|\eta-\gamma| \bar{\rho}(A,\{0\})=|\eta-\gamma| \| A|| .
\end{aligned}
$$

Definition 1.4. (Kuratowski.) Let $\mathscr{C l}$ denote a metric space and let $\mathscr{C}^{*}$ denote the space of all nonvoid, compact subsets of $\mathscr{K}$, metrized by the Hausdorff metric, $\rho$ (cf. Definition 1.3). For a sequence $\left\{A_{i}\right\} \subset \mathscr{C}^{*}, \lim _{i \rightarrow \infty} A_{i}$ is the set of all $x \in \mathscr{C}$ having the property that each neighborhood of $x$ intersects all but a finite number of the $A_{i}$, whereas $\overline{\lim }_{i \rightarrow \infty} A_{i}$ is the set of all $x \in \mathscr{C l}$ having the property that each neighborhood of $x$ intersects infinitely many $A_{i}$. If $\underline{\lim }_{i \rightarrow \infty} A_{i}=\varlimsup_{\lim _{i \rightarrow \infty}} A_{i}$, the common value will be denoted by $\lim _{i \rightarrow \infty} A_{i}$.

Lemma 1.4. ([14, p. 248]) If $\left\{A_{i}\right\} \subset \mathscr{l}^{*}$ and $A \in \mathscr{l}^{*}$, with $\lim _{i \rightarrow \infty} \rho\left(A_{i}, A\right)=0$, then $\lim _{i \rightarrow \infty} A_{i}=A$.

Lemma 1.5. Let $\left\{A_{i}\right\} \subset \mathscr{C}^{*}$ and let $\bar{A} \in \mathscr{C}^{*}$ be a cluster point (in the Hausdorff metric topology) of $\left\{A_{i}\right\}$; then

$$
\lim _{i \rightarrow \infty} A_{i} \subset \bar{A} \subset \varlimsup_{i \rightarrow \infty} A_{i} .
$$


Proof. Let $\left\{A_{i_{k}}\right\}$ satisfy $\lim _{k \rightarrow \infty} \rho\left(A_{i_{k}}, \bar{A}\right)=0$. By [14, pp. 242243]

$$
\lim _{i \rightarrow \infty} A_{i} \subset \lim _{k \rightarrow \infty} A_{i_{k}} \subset \varlimsup_{k \rightarrow \infty} A_{i_{k}} \subset \varlimsup_{i \rightarrow \infty} A_{i} ;
$$

but by Lemma $1.4, \bar{A}=\lim _{k \rightarrow \infty} A_{i_{k}}$.

Corollary 1.2. Let $\left\{A_{i}\right\} \subset \Gamma^{n}$ satisfy $\left\|A_{i}\right\| \leqq \lambda$, for some $\lambda \geqq 0$; if $\bar{A}=\lim _{i \rightarrow \infty} A_{i}$ then $\bar{A} \in \Gamma^{n}$ and $\lim _{i \rightarrow \infty} \rho\left(A_{i}, \bar{A}\right)=0$.

Proof. By Blaschke's Auswahlsatz, the set $U=\left\{A \cap S(0, \lambda) \mid A \in \Gamma^{n}\right\}$ is a compact subset of $\Gamma^{n}$ so that $\left\{A_{i}\right\}$ has a cluster point in $U$. By hypothesis and Lemma 1.5, $\bar{A}$ is the only cluster point of $\left\{A_{i}\right\}$ and then $\bar{A} \in \Gamma^{n}$. Again since $U$ is compact, the assertion of the lemma follows.

Lemma 1.6. Let $\left\{A_{i}\right\} \subset \Omega^{n}$ satisfy, for some $\lambda \geqq 0,\left\|A_{i}\right\| \leqq \lambda$; if $A=\lim A_{i}$ and $A \neq \phi$ then $A \in \Omega^{n}$ and $\lim A_{i}^{*}=A^{*} \in \Gamma^{n}$.

Proof. Since [14, pp. 242-243] $A$ is closed, the fact that $A \in \Omega^{n}$ follows easily from the hypotheses. We shall prove that

$$
A^{*} \equiv\left(\underline{\lim } A_{i}\right)^{*} \subset \underline{\lim } A_{i}^{*} \subset \overline{\lim } A_{i}^{*} \subset\left(\overline{\lim } A_{i}\right)^{*} \equiv A^{*},
$$

the second inequality being trivial. For the proof of the first inequality, let $x \in A^{*}$; by Carathéodory's theorem [7, p. 35] there exist $x^{k} \in A, k=1, \cdots, n+1$, such that $x=\sum_{k=1}^{n+1} \alpha_{k} x^{k}$,

$$
\sum \alpha_{k}=1, \alpha_{k} \geqq 0, k=1, \cdots, n+1 \text {. }
$$

Despite Lemma 1.1, it is trivial to establish that

$$
\{x\}_{r} \equiv\{x\}+S(0, \eta)=\sum_{k=1}^{n+1} \alpha_{k}\left[\left\{x^{k}\right\}+S(0, \eta)\right] \equiv \sum_{k=1}^{n+1} \alpha_{k}\left\{x^{k}\right\}_{\eta_{l}} .
$$

It is easy to see that there exists $K \geqq 0$, independent of $k=1, \cdots, n+1$, such that $\left\{x^{k}\right\}_{\eta} \cap A_{i} \neq \phi$ for all $i \geqq K$. Letting $a_{i}^{k} \in\left\{x^{k}\right\}_{\gamma_{i}} \cap A_{i}$ there follows $\sum_{k=1}^{n+1} \alpha_{k} \alpha_{i}^{k} \in\{x\}_{\eta}$ for all $i \geqq K$; but clearly $\sum_{k=1}^{n+1} \alpha_{k} \alpha_{i}^{k} \in A_{i}^{*}$ and we conclude that $x \in \lim A_{i}^{*}$.

For the proof of the third inequality, let $\bar{x} \in \overline{\lim } A_{i}^{*}$; then by [14, p. 243] there exists a subsequence $\left\{A_{i_{k}}^{*}\right\}$ and a sequence $\left\{x_{k}\right\}$ satisfying $x_{k} \in A_{i_{k}}^{*}$ and $\lim x_{k}=\bar{x}$. Now for each index $k$, there exist vectors $\xi_{k}^{j} \in A_{i_{k}}, j=1, \cdots, n+1$ and numbers $\alpha_{j}^{k} \geqq 0, j=1, \cdots, k+1$, satisfying $\sum_{j=1}^{n+1} \alpha_{j}^{k}=1$ and $x_{k}=\sum_{j=1}^{n+1} \alpha_{j}^{k} \xi_{k}^{j}$. Setting $X_{k}=\left(\xi_{k}^{1}, \cdots, \xi_{k}^{n+1}\right)$ and $\alpha_{k}=\left(\alpha_{1}^{k}, \cdots, \alpha_{n+1}^{k}\right)^{T}$, the superscript denoting transpose, we may write $x_{k}=X_{k} \alpha_{k}$. By virtue of the fact that $\left\|A_{i_{k}}\right\| \leqq \lambda$ for all $k$, it 
is clear that $\left\{X_{k}\right\}$ is contained in a compact subset of the cartesian product $\left(n+1\right.$ factors) $E^{n} \times \cdots \times E^{n}$. Moreover, the compact set $\Sigma=\left\{p \in E^{n+1} \mid p^{i} \geqq 0, \quad i=1, \cdots, n+1 ; \quad \sum_{i=1}^{n+1} p^{i}=1\right\}$ contains $\left\{\alpha_{k}\right\}$. Hence $\left\{X_{k}\right\}$ and $\left\{\alpha_{k}\right\}$ have cluster points $\bar{X}, \bar{\alpha}$ respectively with $\bar{\alpha} \in \Sigma$, and now there follows readily $\bar{x}=\bar{X} \bar{\alpha}$. Writing $\bar{X}=\left(\bar{\xi}^{1}, \cdots, \bar{\xi}^{n+1}\right)$, it is clear that $\bar{\xi}^{j} \in A, j=1, \cdots, n$, so that $\bar{x} \in A^{*}$ and the proof is complete.

\section{Lebesgue measurable functions on $I$ to $Q^{n}$.}

Definition 2.1 (Pliś [16].) A function $F: I \rightarrow \Omega^{n}$ is measurable if and only if the set $E(F, D)=\{t \in I \mid F(t) \cap D \neq \phi\}$ is Lebesgue measurable for each open set $D \subset E^{n}$.

Filippov [9] has stated without proof the following easily established result.

LEMMA 2.1. Let $\mathscr{D}$ be the class of all open balls in $E^{n}$ having positive rational radii and centers with rational coordinates; then a function $F: I \rightarrow \Omega^{n}$ is measurable if and only if the set $E(F, D)$ is measurable for every $D \in \mathscr{D}$.

Lemma 2.2. If $P$ is a closed subset of $I$ and $F: P \rightarrow \Omega^{n}$ is continuous then there exists $\Phi: I \rightarrow \Omega^{n}$ having the following properties:

(i) $\Phi$ is continuous on $I$;

(ii) $\Phi(t)=F(t)$ on $P$;

(iii) for $t \in I,\|\Phi(t)\| \leqq \sup \{\|F(\tau)\| \mid \tau \in P\}$;

(iv) if the range of $F$ is in $\Gamma^{n}$, so is that of $\Phi$.

Proof. Define $\Phi$ on $P$ by setting $\Phi(t)=F(t)$ there; without loss of generality one many assume that $P$ is properly contained in $I$ and that $I$ is the smallest interval containing $P$. If $\left(t_{0}, t_{1}\right)$ is one of the at most countably many complementary intervals of $P$, define $\Phi$ on $\left(t_{0}, t_{1}\right)$ by

$$
\Phi(t)=\left(\frac{t-t_{0}}{t_{1}-t_{0}}\right) F\left(t_{1}\right)+\left(\frac{t_{1}-t}{t_{1}-t_{0}}\right) F\left(t_{0}\right) .
$$

For any points $\tau, \tau_{0}$ in $\left[t_{0}, t_{1}\right]$ there follows

$$
\begin{aligned}
\rho\left(\Phi(\tau), \Phi\left(\tau_{0}\right)\right) & \leqq\left(t_{1}-t_{0}\right)^{-1} \rho\left(\tau\left(F\left(t_{1}\right)-F\left(t_{0}\right)\right), \tau_{0}\left(F\left(t_{1}\right)-F\left(t_{0}\right)\right)\right) \\
& \leqq \frac{\left|\tau-\tau_{0}\right|}{t_{1}-t_{0}}\left\|F\left(t_{1}\right)-F\left(t_{0}\right)\right\|
\end{aligned}
$$

the last inequality being a consequence of Corollary 1.1(v). The 
availability of this estimate makes possible the proof that $\Phi$ is continuous on $I$ by means of an argument like that of Natanson [15, pp. 102-104].

Lemma 2.3. (Plis [16].) If $F: I \rightarrow \Omega^{n}$ is continuous it is measurable.

Filippov [9] has stated the next theorem, without proof, again for bounded functions.

THEOREM 2.1. If $F_{k}: I \rightarrow \Omega^{n}, k=1,2,3, \cdots$, are measurable and if $\lim \rho\left(F_{k}(t), F(t)\right)=0$ almost everywhere (a.e.) on $I$, where $F: I \rightarrow \Omega^{n}$, then $F$ is measurable.

Proof. (After Natanson [15, Th. 2, p. 94].) Let $a, r$ be fixed and such $S^{\circ}(a, r) \in \mathscr{D}$, the class defined in Lemma 2.1, where the superscript denotes interior. For positive integers $m$ satisfying $m r>1$ define

$$
\begin{aligned}
& T_{m}^{k}=E\left(F_{k}, S^{0}\left(a, r-m^{-1}\right)\right), k=1,2,3, \cdots, \\
& Z_{m}^{n}=\bigcap_{k \geqq n} T_{m}^{k}, n=1,2,3, \cdots .
\end{aligned}
$$

We shall prove that

$$
E\left(F, S^{0}(a, r)\right)=\bigcup_{n, m} Z_{m}^{n}
$$

Certainly $T_{m}^{k}$ is measurable by hypothesis and Lemma 2.1; thus $Z_{m}^{n}$ and the right member of (3) are measurable. Then by Lemma 2.1, (3) implies the measurability of $F$.

Let $t_{0} \in E\left(F, S^{0}(a, r)\right)$; then $F\left(t_{0}\right) \cap S^{0}(a, r) \neq \phi$ and there exists an integer $m_{0}, m_{0} r>2$, such that $F\left(t_{0}\right) \cap S^{0}\left(a, r-2 m_{0}^{-1}\right) \neq \phi$. Since $\bar{\rho}\left(F\left(t_{0}\right), F_{k}\left(t_{0}\right)\right) \rightarrow 0$, it follows that $\bar{\rho}\left(F\left(t_{0}\right) \cap S\left(a, r-2 m_{0}^{-1}\right), F_{k}\left(t_{0}\right)\right) \rightarrow 0$. Consequently there exists $n_{0}=n_{0}\left(m_{0}\right)$ such that if $k \geqq n_{0}$ then $F_{k}\left(t_{0}\right) \cap S^{0}\left(a, r-m_{0}^{-1}\right) \neq \phi$. Hence $t_{0} \in T_{m_{0}}^{k}$ for $k \geqq n_{0}$ which implies $t_{0} \in Z_{m_{0}}^{n_{0}}$ and then of course $t_{0} \in \bigcup_{n, m} Z_{m}^{n}$.

Now let $t_{0} \in \bigcup_{n, m} Z_{m}^{n}$; then there exist $n_{0}, m_{0}$ such that $t_{0} \in Z_{m_{0}}^{n_{0}}$. Hence $t_{0} \in T_{m_{0}}^{k}$ for $k \geqq n_{0}$; i.e., $F_{k}\left(t_{0}\right) \cap S^{0}\left(a, r-m_{0}^{-1}\right) \neq \phi$ for $k \geqq n_{0}$. Now since $\bar{\rho}\left(F_{k}\left(t_{0}\right), F\left(t_{0}\right)\right) \rightarrow 0$ it follows that

$$
\bar{\rho}\left(F_{k}\left(t_{0}\right) \cap S\left(\alpha, r-m_{0}^{-1}\right), F\left(t_{0}\right)\right) \rightarrow 0 .
$$

This in turn implies that $S\left(\alpha, r-m_{0}^{-1}\right) \cap F\left(t_{0}\right) \neq \phi$ so that certainly $F\left(t_{0}\right) \cap S^{0}(a, r) \neq \phi$. Thus $t_{0} \in E\left(F, S^{0}(a, r)\right)$ and (3) follows.

The necessity of the condition of the next theorem (generalized Lusin theorem) was established, for bounded, measurable $F$, by Pliś 
[16]. The entire theorem, again restricted to bounded functions, was stated without proof by Filippov [9]. For a measurable set $B \subset I$, let $\mu(B)$ denote its Lebesgue measure.

Theorem 2.2. A function $F: I \rightarrow \Omega^{n}$ is measurable if and only if for each $\eta>0$ there exists $E_{\eta} \subset I$ which is closed, $\mu\left(I-E_{\eta}\right)<\eta$ and the restriction of $F$ to $E_{\eta}$ is continuous.

Proof. (Necessity, using a device of Natanson [15, p. 10].) Let $T_{k}=E\left(F, S^{\sim}(0, k)\right)$, where $k$ is a positive integer and the tilde denotes complementation. Now $\cap T_{k}=\phi$ for otherwise, if $t_{0} \in \bigcap T_{k}$,

$$
F\left(t_{0}\right) \cap S^{\sim}(0, k) \neq \phi
$$

for all $k$, contradicting the assumption that $F\left(t_{0}\right) \in \Omega^{n}$. Hence $\mu\left(\cap T_{k}\right)=$ 0 and since $T_{i} \subset T_{j}$ for $i>j$ it follows that $\lim \mu\left(T_{k}\right)=0$. Thus for $\eta>0$ there exists $k_{0}$ such that $\mu\left(T_{k_{0}}\right)<\eta / 4$; moreover, there exists open $T^{*} \supset T_{k_{0}}$ such that

$$
\mu\left(T^{*}\right)<\mu\left(T_{k_{0}}\right)+\eta / 4<\eta / 2 .
$$

Defining $F^{*}: I \rightarrow \Omega^{n}$ by

$$
\begin{aligned}
& F^{*}(t)=F(t), t \in I-T^{*}, \\
& F^{*}(t)=\{0\}, t \in T^{*},
\end{aligned}
$$

the measurability of $F^{*}$ follows from that of $F$; in addition $\left\|F^{*}(t)\right\| \leqq$ $k_{0}$ for all $t \in I$. Hence, by the aforementioned theorem of Plis [16], there exists closed $E_{\eta}^{*} \subset I$ such that the restriction of $F^{*}$ to $E_{\eta}^{*}$ is continuous and $\mu\left(I-E_{\eta}^{*}\right)<\eta / 2$. Consequently, the restriction of $F$ to the set $E_{\eta} \equiv\left(I-T^{*}\right) \bigcap E_{\eta}^{*}$ is continuous and $E_{\eta}$ is certainly closed. Moreover,

$$
\mu\left(I-E_{\eta}\right)=\mu\left(T^{*} \mathrm{U}\left(I-E_{\eta}^{*}\right)\right) \leqq \mu\left(T^{*}\right)+\mu\left(I-E_{\eta}^{*}\right)<\eta,
$$

and the argument is complete.

(Sufficiency.) For each $\eta>0$, denote by $\Phi(\circ, \eta)$ the continuous extension of $F$, from $E_{\eta}$ to $I$, guaranteed by Lemma 2.2. Let $\eta_{m}=2^{-m}, m=1,2,3, \cdots ;$ then setting

$$
S_{m}=I-E_{\eta_{m}}
$$

it follows that $\mu\left(S_{m}\right)<2^{-m}$. Define

$$
M_{i}=\bigcup_{k \geqq i} S_{k} ; Q=\bigcap_{i \geqq 1} M_{i} .
$$

Now $M_{1} \supset M_{2} \supset \cdots$ so that $\lim \mu\left(M_{i}\right)=\mu(Q)$; but since $\mu\left(M_{i}\right)<\sum_{k=i}^{\infty} 2^{-k}$ there follows $\mu(Q)=0$. Let $t_{0} \in I-Q$; then $t_{0} \in \bigcup_{i \geq 1}\left(I-M_{i}\right)$ so that 
$t_{0} \in I-M_{i_{0}}$ for some $i_{0}$. But then $t_{0} \in I-S_{k}$ for all $k \geqq i_{0}$; i.e., $\rho\left(F\left(t_{0}\right), \Phi\left(t_{0}, \eta_{k}\right)\right)=0$ for all $k \geqq i_{0}$ and this in turn implies

$$
\lim \rho\left(F\left(t_{0}\right), \Phi\left(t_{0}, \eta_{k}\right)\right)=0 \text {. }
$$

By Lemma 2.3, $\Phi\left(\circ, \eta_{k}\right)$ is measurable for each $k$ so that by Theorem 2.1 and the result just obtained, $F$ is measurable.

CoRollaRY 2.1. If $F: I \rightarrow \Omega^{n}$ is continuous (measurable) then the function $F^{*}: I \rightarrow \Gamma^{n}$ defined by $F^{*}(t)=(F(t))^{*}$ is continuous (measurable).

Proof. The assertion concerning continuity is immediate from Lemma 1.3 (ii). Now suppose $F$ is measurable; by Theorem 2.2, for $\eta>0$ there exists closed $E_{\eta} \subset I$ such that $\mu\left(I-E_{\eta}\right)<\eta$ and the restriction of $F$ to $E_{\eta}$ is continuous. But by Lemma 1.3 (ii), the restriction of $F^{*}$ to $E_{\eta}$ is continuous. Another application of Theorem 2.2 yields the measurability of $\mathrm{F}^{*}$.

The next two lemmas were originally stated for bounded functions; an examination of their proofs (vide [12]) reveals, in the light of Theorem 2.2, that this boundedness restriction is superfluous.

Lemma 2.4. (Hermes-Filippov.) Let $g: E^{n} \rightarrow E^{k}$ be continuous and let $H: I \rightarrow \Omega^{n}$ be measurable. If $r: I \rightarrow E^{n}$ is measurable and $r(t) \in g(H(t))$ on $I$ then there exists measurable $\nu: I \rightarrow E^{n}$ satisfying $\nu(t) \in H(t)$ and $r(t)=g(\nu(t))$ on $I$.

Lemma 2.5. (Hermes.) Let $R: I \rightarrow \Omega^{n}$ be measurable and let $w: I \rightarrow E^{n}$ be measurable; then there exists measurable $r: I \rightarrow E^{n}$ satisfying $r(t) \in R(t)$ and $\|w(t)-r(t)\|=\alpha(w(t), R(t))$ on $I$.

The next lemma was originally stated by Hermes [11, Lemma 1.1] for bounded functions; again by virtue of Theorem 2.2, the boundedness restriction is superfluous. A function $F: I \rightarrow \Omega^{n}$ is approximately continuous at $t \in I$ if and only if there exists a measurable set $B \subset I$ for which $t$ is a point of density and such that the restriction of $F$ to $B$ is continuous at $t$.

Lemma 2.6. If $F: I \rightarrow \Omega^{n}$ is measurable then $F$ is approximately continuous a.e. on $I$.

Definition 2.2. (i ) Let $F: I \rightarrow \Omega^{n}$; if there exists a Lebesgue summable function $h: I \rightarrow E^{1}$ such that $\|F(t)\| \leqq h(t)$ on $I$ then $F$ is integrably bounded. 
(ii) Let $A$ be an index set and let $F_{\gamma}: I \rightarrow \Omega^{n}$ for all $\gamma \in A$; if there exists a Lebesgue summable function $h: I \rightarrow E^{1}$ such that $\left\|F_{\gamma}(t)\right\| \leqq h(t)$ for all $t \in I$ and all $\gamma \in A$ then $\left\{F_{\gamma} \mid \gamma \in A\right\}$ is uniformly integrably bounded.

The next lemma has an easy proof which will be omitted.

Lemma 2.7. (i) If $F: I \rightarrow Q^{n}$ is continuous it is integrably bounded.

(ii) If $F: I \rightarrow \Omega^{n}$ is integrably bounded then the function $F^{*}$ defined in Corollary 2.1 has the same integrable bound as $F$.

Definition 2.3. Let $F: I \rightarrow Q^{n}$ be such that for each $t \in I$ the function $\rho(F(\circ), F(t))$ is summable on $I$. A point $t \in I$ for which

$$
\lim _{\eta \rightarrow 0} \eta^{-1} \int_{t}^{t+\eta} \rho(F(\tau), F(t)) d \tau=0
$$

is called a Lebesgue point of $F$.

THeOREM 2.3. If $F: I \rightarrow \Omega^{n}$ is measurable and integrably bounded then almost all $t \in I$ are Lebesgue points of $F$.

Proof. Theorem 2.2 and the continuity of $\rho(\circ, \circ)$, together with Lusin's theorem for real valued functions, implies that $\rho(F(\circ), F(t))$ is measurable for each $t \in I$. Let $h$ be an integrable bound for $F$; without loss of generality one may suppose that $h(t)>0$ on $I$. By Corollary 1.1(iv), $\rho(F(\tau), F(t)) \leqq h(\tau)+h(t)$ for all $\tau, t \in I$. Hence $\rho(F(\circ), F(t))$ is summable on $I$ for each $t \in I$. Now by Lemma 2.6 and [15, Th. 5, p. 255] almost all points of $I$ are, at once, points of approximate continuity of $F$ and Lebesgue points of $h$. Let $t$ be such a point and let $B \subset I$ be a measurable set for which $t$ is a point of density and such that the restriction of $F$ to $B$ is continuous at t. For $\eta>0$, set

$$
\begin{aligned}
& B_{1}(\eta)=[t, t+\eta] \cap B, \\
& B_{2}(\eta)=[t, t+\eta] \cap(I-B) .
\end{aligned}
$$

Then, given $\varepsilon>0$, one may choose $\eta=\eta(\varepsilon, t)>0$ sufficiently small that the following three conditions are satisfied:

(i) for $\tau \in B_{1}(\eta), \rho(F(\tau), F(t))<\varepsilon / 6$;

(ii) $\mu\left(B_{2}(\eta)\right)<\varepsilon \eta / 6 h(t)$;

(iii) $\int_{t}^{t+\eta}|h(\tau)-h(t)| d \tau<\eta \varepsilon / 3$.

By virtue of (i), (ii), (iii) and Corollary 1.1 (iv) there follows 


$$
\begin{aligned}
\eta^{-1} \int_{t}^{t+\eta} \rho(F(\tau), F(t)) d \tau & =\eta^{-1} \int_{B_{1}(\eta)} \rho(F(\tau), F(t)) d \tau+\eta^{-1} \int_{B_{2}(\eta)} \rho(F(\tau), F(t)) d \tau \\
& <\varepsilon / 3+\eta^{-1} \int_{B_{2}(\eta)}[\|F(\tau)\|+\|F(t)\|] d \tau \\
& <\varepsilon / 3+\eta^{-1} \int_{t}^{t+\eta}|h(\tau)-h(t)| d \tau+2 h(t) \eta^{-1} \mu\left(B_{2}(\eta)\right) \\
& <\varepsilon / 3+\varepsilon / 3+\varepsilon / 3=\varepsilon .
\end{aligned}
$$

Thus $\lim _{\eta \rightarrow 0+} \eta^{-1} \int_{t}^{t+\eta} \rho(F(\tau), F(t)) d \tau=0$, and a similar argument shows that the left hand limit is also zero.

We close this section with the following important lemma on the measurability of composite functions.

Lemma 2.8. Let $D$ be a nonvoid, open subset of $E^{1} \times E^{n}$ and let $R: E^{1} \times E^{n} \rightarrow \Omega^{n}$ satisfy:

(i) for each $t$ in the projection of $D$ on $E^{1}, R(t, \circ)$ is continuous on the set $D_{t}=\left\{x \in E^{n} \mid(t, x) \in D\right\}$;

(ii) for each $x$ in the projection of $D$ on $E^{n}$ and each compact interval $I \subset E^{1}$ for which $I \times\{x\} \subset D, R(\circ, x)$ is measurable on $I$;

(iii) for each compact $C \subset D$ there exists a Lebesgue summable function $h_{c}: E^{1} \rightarrow E^{1}$ such that $\|R(t, x)\| \leqq h_{c}(t)$ on $C$.

If $I$ is a compact interval in $E^{1}$ and $S$ is a compact ball in $E^{n}$ satisfying $I \times S \subset D$ then for each continuous function $x: I \rightarrow S$ the function $R(\circ, x(\circ))$ is integrably bounded and measurable on $I$.

Proof. If the assertion of the lemma is true with "continuous" replaced by "step" as the restriction on $x: I \rightarrow S$ then the validity of the original statement, insofar as measurability is concerned, follows by virtue of (i) and Theorem 2.1 since a continuous function $x: I \rightarrow S$ may be uniformly approximated by step functions. Hence suppose that for $c_{k} \in S, k=1, \cdots, m, x^{*}: I \rightarrow S$ is defined by

$$
x *(t)=c_{k}, t \in I_{k}, k=1, \cdots, m,
$$

where $I=\bigcup I_{k}, I_{j} \cap I_{k}=\phi$ for $j \neq k$ and each $I_{k}$ is an interval. Then for an open set $K \subset E^{n}, E\left(R\left(\circ, x^{*}(\circ)\right), K\right)=\bigcup M_{j}$,

$$
M_{j}=\left\{t \in I_{j} \mid R\left(t, c_{j}\right) \cap K \neq \phi\right\}, j=1, \cdots, m .
$$

But by (ii), each $M_{j}$ is measurable so that $E\left(R\left(\circ, x^{*}(\circ)\right), K\right)$ is measurable. Integrable boundedness of $R(\circ, x(\circ))$ is an easy consequence of (iii).

3. Trajectory integrals of measurable functions. In this 
section we set $I=[0,1]$ without loss of generality and suppose that $F: I \rightarrow \Omega^{n}$ is a given function. As in the introduction we denote by $\mathscr{F}_{I}(F)$ the set of all Lebesgue summable functions $u: I \rightarrow E^{n}$ having the property that $u(t) \in F(t)$ a.e. on $I$. Let $\mathscr{T}$ on $\mathscr{L}_{1}^{n}(I)$ be defined by

$$
(\mathscr{T} q)(t)=\int_{0}^{t} q(\tau) d \tau, t \in I,
$$

and define

$$
\mathscr{S}_{I}(F)=\mathscr{T} \mathscr{F}_{I}(F) .
$$

$\mathscr{S}_{I}(F)$ may be considered as a subset of any of a number of Banach spaces but the ones we shall be primarily concerned with here are $\mathscr{C}^{n}(I)$ and $\mathscr{N}_{\mathscr{A}} \mathscr{C}^{n}(I)$.

Lemma 3.1. (i) If $F: I \rightarrow \Omega^{n}$ is measurable and integrably bounded then $\mathscr{F}_{I}(F) \neq \phi$.

(ii) If $F: I \rightarrow \Gamma^{n}$ then $\mathscr{F}_{I}(F)$ is a convex subset of $\mathscr{L}_{1}^{n}(I)$.

Proof. That there exists a measurable $\nu: I \rightarrow E^{n}$ satisfying $\nu(t) \in F(t)$ a.e. on $I$ follows from Lemma 2.4 by taking $g=0, r=0$, and $H=F$. The assertion of (i) then follows by the integrable boundedness of $F$. The proof of (ii) is trivial.

THEOREM 3.1. If $F: I \rightarrow \Gamma^{n}$ is measurable and integrably bounded then $\mathscr{S}_{I}(F) \in \mathscr{K}^{n}(I)$; moreover, $\mathscr{S}_{I}(F)$ is a weakly compact subset of $\operatorname{ASC}^{n}(I)$.

Proof. From Lemma 3.1 and the linearity of $\mathscr{T}$ follow the facts that $\mathscr{S}_{I}(F)$ is nonvoid and convex; that $\mathscr{S}_{I}(F)$ is conditionally compact follows readily from the integrable boundedness of $F$ together with the Arzelà-Ascoli theorem. The first assertion of the theorem will be established if we show that $\mathscr{S}_{I}(F)$ is closed in $\mathscr{C}^{n}(I)$. To this end let $w \in \overline{\mathscr{S}_{I}(F)}$ and let $\left\{w_{m}\right\} \subset \mathscr{S}_{I}(F)$ satisfy $\lim \left\langle w_{m}-w\right\rangle=0$. Now $\dot{w}_{m}(t) \in F(t)$ a.e. on $I$ so that with $h$ denoting the integrable bound on $F$ we obtain

$$
\begin{aligned}
\left\|w\left(t_{2}\right)-w\left(t_{1}\right)\right\| \leqq & \left\|w\left(t_{2}\right)-w_{m}\left(t_{2}\right)\right\|+\left\|w\left(t_{1}\right)-w_{m}\left(t_{1}\right)\right\| \\
& +\left\|w_{m}\left(t_{2}\right)-w_{m}\left(t_{1}\right)\right\|<\varepsilon+\left|\int_{t_{1}}^{t_{2}} h(\tau) d \tau\right|
\end{aligned}
$$

for $\varepsilon>0$ and $m$ sufficiently large. Thus $w$ is absolutely continuous on $I$ and it is easy to see that there exists measurable $U \subset I, \mu(I-U)=$ 0 , having the following properties:

(i) $\dot{w}(t)$ exists on $U$;

(ii) each $t \in U$ is a Lebesgue point of $F$. 
The validity of (ii) is of course a consequence of Theorem 2.3. With $\nu$ being the function defined in Definition 1.3, by virtue of Theorem 2.2, the Lusin theorem for real valued functions and the continuity of $\nu(\circ, \circ)$ on $\Gamma^{n} \times E^{n}$ [3, Lemma 1] there follows the fact that $\nu(F(\circ), p)$ is measurable for each $p \in E^{n}$. By virtue of Lemma 1.2 (iii) and Corollary 1.1 (iv) there obtains $|\nu(F(t), p)| \leqq h(t)$ for all $(t, p) \in I \times E^{n}$ and thus $\nu(F(\circ), p)$ is summable for $p \in E^{n}$. Moreover, there exists measurable $V \subset I, \mu(I-V)=0$, such that for all $(t, p) \in V \times E^{n}$ and all $m$,

$$
\dot{w}_{m}(t) \circ p \leqq \nu(F(t), p) .
$$

Thus for all $m$, all $p \in E^{n}$ and all $t_{1}, t_{2} \in I$,

$$
\left[w_{m}\left(t_{2}\right)-w_{m}\left(t_{1}\right)\right] \circ p \leqq \int_{t_{1}}^{t_{2}} \nu(F(\tau), p) d \tau ;
$$

in particular for $t \in U, \eta>0$, all $m$ and all $p$ such that $\|\mathbf{p}\|=1$,

$$
\begin{aligned}
\eta^{-1}\left[w_{m}(t+\eta)-w_{m}(t)\right] \circ p & \leqq \eta^{-1} \int_{t}^{t+\eta} \nu(F(\tau), p) d \tau \\
& \leqq \nu(F(t), p)+\eta^{-1} \int_{t}^{t+\eta} \rho(F(\tau), F(t)) d \tau,
\end{aligned}
$$

the final inequality being a consequence of Lemma 1.2(iii). For all $\eta>0$ such that $t+\eta \in I$, the convergence of $w_{m}$ to $w$ implies that

$$
\eta^{-1}[w(t+\eta)-w(t)]=\lim _{m \rightarrow \infty} \eta^{-1}\left[w_{m}(t+\eta)-w_{m}(t)\right] .
$$

This and the last formula line imply that for $\|p\|=1, t \in U, \eta>0$ and $t+\eta \in I$,

$$
\eta^{-1}[w(t+\eta)-w(t)] \circ p \leqq \nu(F(t), p)+\eta^{-1} \int_{t}^{t+\eta} \rho(F(\tau), F(t)) d \tau .
$$

Letting $\eta \rightarrow 0+$ in this inequality yields, for $\|p\|=1$,

$$
\dot{w}(t) \circ p \leqq \nu(F(t), p)
$$

and in turn this implies $\left[19\right.$, Th. 5.3] that $\dot{w}(t) \in F(t)$. Thus is $\mathscr{S}_{I}(F)$ closed.

For the proof of the second assertion of the theorem, let $x$ be a weak limit point (i.e., a limit point relative to the weak topology in $\left.\mathscr{N} \mathscr{A} \mathscr{C}^{n}(I)\right)$ of $\mathscr{S}_{I}(F)$. By [6, IV. 13.31] there exists a sequence $\left\{x_{m}\right\} \subset \mathscr{S}_{I}(F)$ which converges pointwise to $x$ on $I$. But by the first assertion of the theorem, there is a subsequence $\left\{x_{m_{k}}\right\}$ which converges in $\mathscr{C}^{n}(I)$ to $x$ so that necessarily $x \in \mathscr{S}_{I}(F)$. Thus is $\mathscr{S}_{I}(F)$ weakly closed. Now $\left\|\int_{E} q(\tau) d \tau\right\| \leqq \int_{E} h(\tau) d \tau$ for all $q \in \mathscr{F}_{I}(F)$ and all measurable $E \subset I$; hence by [6, IV. 8.11] and the absolute continuity of the set 
function $\int_{E} h(\tau) d \tau, \mathscr{F}_{I}(F)$ is weakly sequentially compact in $\mathscr{L}_{1}^{n}(I)$. Since $\mathscr{T}$ is linear and continuous with respect to the metric topologies in $\mathscr{L}_{1}^{n}(I)$ and $\mathscr{N} \mathscr{A} \mathscr{C}^{n}(I)$, by [6, V. 3.15] $\mathscr{S}_{I}(F)$ is weakly sequentially compact in $\mathscr{N} \mathscr{A} \mathscr{C}^{n}(I)$. Now the weak compactness of $\mathscr{S}_{I}(F)$ is a consequence of $[6, \mathrm{~V} .6 .1]$.

THEOREM 3.2. Let $F, F_{k}: I \rightarrow \Gamma^{n}, k=1,2,3, \cdots$, satisfy

$$
\lim \rho\left(F_{k}(t), F(t)\right)=0
$$

on $I$; if $\left\{F_{k}\right\}$ is uniformly integrably bounded and each $F_{k}$ is measurable then $\mathscr{S}_{I}\left(F_{k}\right)$ and $\mathscr{S}_{I}(F)$ are in $\mathscr{K}^{n}(I)$ and $\lim \sigma\left(\mathscr{S}_{I}\left(F_{k}\right), \mathscr{S}_{I}(F)\right)=0$.

Proof. That $\mathscr{S}_{I}\left(F_{k}\right) \in \mathscr{K}^{n}(I)$ is a consequence of Theorem 3.1. That $F$ is measurable is implied by Theorem 2.1. Let $h$ be a uniform integrable bound for $\left\{F_{k}\right\}$ and let $t \in I$ be fixed; by hypothesis and Corollary 1.1 (iv) we find that, given $\varepsilon>0$, there exists $K=K(\varepsilon, t)$ such that for $k>K,\|F(t)\|<\varepsilon+\left\|F_{k}(t)\right\| \leqq \varepsilon+h(t)$. Thus $F$ is integrably bounded by $h$ and from Theorem 3.1 there follows $\mathscr{S}_{I}(F) \in \mathscr{K}^{n}(I)$. Now there exists $w_{k} \in \mathscr{S}_{I}\left(F_{k}\right)$ such that $\beta\left(w_{k}, \mathscr{S}_{I}(F)\right)=$ $\bar{\sigma}\left(\mathscr{S}_{I}\left(F_{k}\right), \mathscr{S}_{I}(F)\right)$. Let $q_{k} \in \mathscr{F}_{I}\left(F_{k}\right)$ be such that $w_{k}=\mathscr{\mathscr { T }} q_{k}$ and, by Lemma 2.5, let $u_{k} \in \mathscr{F}_{I}(F)$ satisfy $\left\|u_{k}(t)-q_{k}(t)\right\|=\alpha\left(q_{k}(t), F(t)\right) \leqq$ $\bar{\rho}\left(F_{k}(t), F(t)\right)$ on $I$. Then $\bar{\sigma}\left(\mathscr{S}_{I}\left(F_{k}\right), \mathscr{S}_{I}(F)\right) \leqq\left\langle w_{k}-\mathscr{T} u_{k}\right\rangle$; but

$$
\left\langle w_{k}-\mathscr{T} u_{k}\right\rangle \leqq \int_{0}^{1}\left\|q_{k}(\tau)-u_{k}(\tau)\right\| d \tau=\int_{0}^{1} \alpha\left(q_{k}(\tau), F(\tau)\right) d \tau
$$

and since $\alpha\left(q_{k}(t), F(t)\right) \rightarrow 0$ on $I$ and $\alpha\left(q_{k}(t), F(t)\right) \leqq 2 h(t)$ on $I$ it follows from [6, III. 6.16] that $\lim \left\langle w_{k}-\mathscr{T} u_{k}\right\rangle=0$. Hence

$$
\lim \bar{\sigma}\left(\mathscr{S}_{I}\left(F_{k}\right), \mathscr{S}_{I}(F)\right)=0 \text {. }
$$

There also exists $y_{k} \in \mathscr{S}_{I}(F)$ such that $\beta\left(y_{k}, \mathscr{S}_{I}\left(F_{k}\right)\right)=\bar{\sigma}\left(\mathscr{S}_{I}(F), \mathscr{S}_{I}\left(F_{k}\right)\right)$. Let $u_{k} \in \mathscr{F}(F)$ satisfy $y_{k}=\mathscr{T} u_{k}$ and, by Lemma 2.5, let $q_{k} \in \mathscr{F}_{I}\left(F_{k}\right)$ satisfy $\quad\left\|\mathrm{u}_{k}(t)-q_{k}(t)\right\|=\alpha\left(u_{k}(t), F_{k}(t)\right) \leqq \bar{\rho}\left(F(t), F_{k}(t)\right) \quad$ on $\quad I$. Then $\bar{\sigma}\left(\mathscr{S}_{I}(F), \mathscr{S}_{I}\left(F_{k}\right)\right) \leqq\left\langle y_{k}-\mathscr{T} q_{k}\right\rangle ;$ but

$$
\left\langle y_{k}-\mathscr{T} q_{k}\right\rangle \leqq \int_{0}^{1}\left\|u_{k}(\tau)-q_{k}(\tau)\right\| d \tau=\int_{0}^{1} \alpha\left(u_{k}(\tau), F_{k}(\tau)\right) d \tau .
$$

Arguing as in the preceding part of the proof we conclude

$$
\lim \bar{\sigma}\left(\mathscr{S}_{I}(F), \mathscr{S}_{I}\left(F_{k}\right)\right)=0
$$

and the proof is complete.

Definition 3.1. Let $\mathscr{S}$ be a set of functions on $I$ to $E^{n}$; then

$$
G(t ; \mathscr{S})=\{\varphi(t) \mid \varphi \in S\}, t \in I .
$$


LEMMA 3.2. If either of the following conditions is satisfied then for all $t \in I, G(t ; \mathscr{S}) \in \Gamma^{n}$ :

(i ) $\mathscr{S} \in \mathscr{K}^{n}(I)$;

(ii) $\mathscr{S}$ is a nonvoid, convex, weakly compact subset of $\operatorname{NSAC}^{n}(I)$.

Proof. (i) is an immediate consequence of [4, Th. 1.4]. For (ii) we observe first of all that by [6, IV. 12.3] there is a unique nonvoid, convex, weakly compact subset $\mathscr{F} \subset \mathscr{L}_{1}^{n}(I)$ such that $\mathscr{S}=\mathscr{T} \mathscr{F}$. By virtue of $[6, \mathrm{~V}, 6.1], \mathscr{F}$ is weakly sequentially compact; from [6, IV. 8.8] it then follows that $F$ is bounded. The function $\mathscr{T}_{t}: \mathscr{L}_{1}^{n}(I) \rightarrow E^{n}$ defined for each fixed $t \in I$ by

$$
\mathscr{T}_{t} q=\int_{0}^{t} q(\tau) d \tau
$$

is linear and continuous with respect to the metric topologies in $\mathscr{L}_{1}^{n}(I), E^{n}$; hence by [6, V. 3.15] it is continuous with respect to the weak topologies in these spaces. Consequently $\mathscr{T}_{t} \mathscr{F}$ is bounded, convex and weakly compact, hence, by [6, V. 3.13], closed. We conclude that $G(t ; \mathscr{S}) \equiv \mathscr{T}_{t} \mathscr{F} \in \Gamma^{n}$.

The next lemma generalizes a result due to Hermes [12, Th. 1.2].

Lemma 3.3. If $F: I \rightarrow \Omega^{n}$ is measurable and integrably bounded then $G\left(t ; \mathscr{S}_{I}(F)\right)=G\left(t ; \mathscr{S}_{I}\left(F^{*}\right)\right) \in \Gamma^{n}$ for all $t \in I$.

Proof. By Corollary 2.1, Lemma 2.7 (ii), Theorem 3.1 and Lemma 3.2, $G\left(t ; \mathscr{S}_{I}\left(F^{*}\right)\right) \in \Gamma^{n}$. Certainly $G\left(t ; \mathscr{S}_{I}(F)\right) \subset G\left(t ; \mathscr{S}_{I}\left(F^{*}\right)\right)$ and the remainder of the proof coincides with the second part of Hermes' proof for [12, Th. 1.2].

Hermes [11] has observed that: if $F: I \rightarrow \Omega^{n}$ is Borel measurable [1] then it is measurable. Our next result is the combined assertion of Theorems 1 through 4 of [1] for Borel measurable, integrably bounded $F: I \rightarrow \Omega^{n}$. It is an immediate consequence of Lemma 3.3 and Hermes' observation.

Corollary 3.1. If $F: I \rightarrow \Omega^{n}$ is Borel measurable and integrably bounded then for each $t \in I, G\left(t ; \mathscr{S}_{I}(F)\right) \in \Gamma^{n}$.

Lemma 3.3 provides the instrument for establishing the following corollaries to Theorem 3.2.

CoRollary 3.2. Let $F, F_{k}: I \rightarrow \Omega^{n}, k=1,2,3, \cdots$, satisfy

$$
\lim \rho\left(F_{k}(t), F(t)\right)=0
$$


on $I$; if $\left\{F_{k}\right\}$ is uniformly integrably bounded and each $F_{k}$ is measurable then for each $t \in I, G\left(t ; \mathscr{S}_{I}\left(F_{k}\right)\right)$ and $G\left(t ; \mathscr{S}_{I}(F)\right)$ are in $\Gamma^{n}$ and

$$
\lim \rho\left(G\left(t ; \mathscr{S}_{I}\left(F_{k}\right)\right), G\left(t ; \mathscr{S}_{I}(F)\right)\right)=0,
$$

uniformly on $I$.

Proof. By Corollary 2.1 and Lemma 2.7, each $F_{k}^{*}$ is measurable and $\left\{F_{k}^{*}\right\}$ has the same uniform integrable bound as $\left\{F_{k}\right\}$. By Theorem 2.1, $F$ is measurable and, by an argument like that used in Theorem 3.2, $F$ is integrably bounded. Thus by Corollary 2.1 and Lemma 2.7, $F^{*}$ is measurable and integrably bounded and, by hypothesis and Lemma $1.3(\mathrm{ii}), \quad \lim \rho\left(F_{k}^{*}(t), F^{*}(t)\right)=0$. From Theorem 3.2 there follows $\lim \sigma\left(\mathscr{S}_{I}\left(F_{k}^{*}\right), \mathscr{S}_{I}\left(F^{*}\right)\right)=0$ and this result together with [4, Th. 1.5] implies

$$
\lim \rho\left(G\left(t ; \mathscr{S}_{I}\left(F_{k}^{*}\right)\right), G\left(t ; \mathscr{S}_{I}\left(F^{*}\right)\right)\right)=0,
$$

uniformly for $t \in I$. The proof is completed by application of Lemma 3.3.

CoRollaRy 3.3. Let $F_{k}: I \rightarrow \Omega^{n}, k=1,2,3, \cdots$, satisfy the following conditions:

(i) $\left\{F_{k}\right\}$ is uniformly integrably bounded;

(ii) for each $k, F_{k}$ is Borel measurable;

(iii) $F(t)=\lim F_{k}(t)$ exists and is nonvoid for each $t \in I$. Then $F: I \rightarrow \Omega^{n}$ and, for each $t \in I$,

$$
\lim G\left(t ; \mathscr{S}_{I}\left(F_{k}\right)\right)=G\left(t ; \mathscr{S}_{I}(F)\right) \in \Gamma^{n} .
$$

Proof. By virtue of (i), (iii) and Lemma 1.6, $F: I \rightarrow \Omega^{n}$ and $\lim F_{k}^{*}(t)=F^{*}(t)$. Lemma 2.7 implies that $\left\{F_{k}^{*}\right\}$ has the same uniform integrable bound as $\left\{F_{k}\right\}$ so that Corollary 1.2 yields $\lim \rho\left(F_{k}^{*}(t), F^{*}(t)\right)=$ 0 . The observation of Hermes quoted above, together with (ii) and Corollary 2.1, yields the measurability of $F_{k}^{*}$. Now Corollary 3.2 and Lemma 1.4 permit the assertion

$$
\lim G\left(t ; \mathscr{S}_{I}\left(F_{k}^{*}\right)\right)=G\left(t ; \mathscr{S}_{I}\left(F^{*}\right)\right) \in \Gamma^{n} ;
$$

hence Lemma 3.3 yields

$$
\lim G\left(t ; \mathscr{S}_{I}\left(F_{k}\right)\right)=G\left(t ; \mathscr{S}_{I}\left(F^{*}\right)\right) \in \Gamma^{n} .
$$

But the assertion of [1, Th. 5] is that the left member of this equation is equal to $G\left(t ; \mathscr{S}_{I}(F)\right)$; the proof is complete.

Discussion. It is easy to see that in Corollary 3.3 , the requirement that $F_{k}$ be nonvoid, compact valued for each $k$ can be replaced 
by the requirement that it be nonvoid, closed valued for each $k$. The corresponding replacement can be made in Corollary 3.1. It is noteworthy that Corollary 3.1 bears out the anticipation, expressed in the introduction that a study of $\mathscr{S}_{I}(F)$ subsumes, in an obvious sense, a study of Aumann's integral. Corollary 3.3 shows that our expectations in this direction cannot be too high; indeed, under hypotheses of this corollary, ( $)$ appears to be the strongest result we can obtain within the confines of the theory developed in this paper. The utilization of [1, Th. 5] in this corollary could be supplanted by a counterpart of Theorem 2.1 in which Hausdorff convergence is replaced by Kuratowski convergence. However, we have not been successful in obtaining such a counterpart of Theorem 2.1; moreover, in view of the proof of Theorem 2.1 it does not appear likely that such a counterpart is valid. It is also noteworthy that the lack of such a counterpart for Theorem 2.1 prevents the inference from [1, Th. 5] alone that $G\left(t ; \mathscr{S}_{I}(F)\right) \neq \phi$ for some $t \in I$ even under the hypotheses of Corollary 3.3.

The weak compactness of $\mathscr{S}_{I}(F)$ in $\mathscr{N} \mathscr{A} \mathscr{C}^{n}(I)$ may be shown to follow directly from the hypotheses of Theorem 3.1; the device of using the compactness of $\mathscr{S}_{I}(F)$ in $\mathscr{C}^{n}(I)$ to establish weak compactness of $\mathscr{S}_{I}(F)$ was a matter of convenience in the proof of that theorem. Taking this observation into account, it is not difficult to see that Corollary 3.2 may be established independently by means of an argument which depends only on weak compactness of $\mathscr{S}_{I}(F)$, Lemma 3.2 (ii), Lemma 3.3 and Lemma 2.5. Thus Corollaries 3.1, 3.2 constitute a theory which is a direct counterpart of Aumann's theory, the major distinction between the two theories being that between Hausdorff and Kuratowski convergence. The discussion of the preceding paragraph indicates that whereas these theories are supplementary, neither implies the other.

The proof of [12, Corollary 1.1] applies with trivial modification, taking into account Lemma 3.3, to yield

Lemma 3.4. Let $F: I \rightarrow \Omega^{n}$ be measurable and integrably bounded, and let $y \in \mathscr{S}_{I}\left(F^{*}\right)$; then for each $\eta>0$ there exists $z_{\eta} \in \mathscr{S}_{I}(F)$ satisfying $\left\langle y-z_{\eta}\right\rangle<\eta$.

This lemma has the following immediate consequence.

CoRollary 3.4. If $F: I \rightarrow \Omega^{n}$ is measurable and integrably bounded then $\mathscr{S}_{I}\left(F^{*}\right)$ is the closure of $\mathscr{S}_{I}(F)$ in $\mathscr{C}^{n}(I)$.

REMARK 3.1. [12, Example 2.3.] shows that with the hypotheses 
of Corollary 3.4 $\mathscr{S}_{I}(F)$ need not be closed in $\mathscr{C}^{n}(I)$; there thus appears to be no possibility of generalizing Theorem 3.2 by requiring that $F, F_{k}$ have values in $\Omega^{n}$.

Let us denote by $\mathscr{S}_{I}^{*}(F)$ the closed (in $\mathscr{N} \mathscr{A} \mathscr{C}^{n}(I)$ ) convex hull of $\mathscr{S}_{I}(F)$ and by $\mathscr{S}_{I}^{\sharp}(F)$, the weak closure of $\mathscr{S}_{I}(F)$ in $\mathscr{N} \mathscr{A} \mathscr{C}^{n}(I)$.

THEOREM 3.3. If $F: I \rightarrow \Omega^{n}$ is measurable and integrably bounded then

$$
\mathscr{S}_{I}^{*}(F)=\mathscr{S}_{I}^{*}(F)=\mathscr{S}_{I}\left(F^{*}\right) \text {. }
$$

Proof. By means of an argument like that for the second assertion of Theorem 3.1 it may be inferred that $\mathscr{S}_{I}(F)$ is weakly sequentially compact. Now there follows from [6, V. 3.13, 3.14] and Theorem 3.1,

$$
\mathscr{S}_{I}^{*}(F) \subset \mathscr{S}_{I}^{*}(F) \subset \mathscr{S}_{I}\left(F^{*}\right) \text {. }
$$

But from these inclusions, Lemma 3.4 and [6, IV. 13.31], the theorem follows.

REMARK 3.2. It is easy to see that $\mathscr{S}_{I}^{*}(F)=\mathscr{F}_{F}^{*}(F)$, where $\mathscr{F}_{I}{ }^{*}(F)$ is the closed convex hull of $\mathscr{F}_{I}(F)$.

Arguing again as in the proof of the second assertion of Theorem 3.1 , it follows that if $F: I \rightarrow Q^{n}$ is measurable and integrably bounded and if $\mathscr{S}_{I}(F)$ is closed in $\mathscr{C}^{n}(I)$ then $\mathscr{S}_{I}(F)$ is weakly closed in $\operatorname{TSAC}^{n}(I)$.

In view of this result, Theorem 3.3 yields

COROLlaRY 3.5. If $F: I \rightarrow \Omega^{n}$ is measurable and integrably bounded then $\mathscr{S}_{I}(F) \in \mathscr{K}^{n}(I)$ only if $\mathscr{S}_{I}(F)=\mathscr{S}_{I}\left(F^{*}\right)$.

The final result of this section provides a marked strengthening of Theorem 3.1 and of the assertion of Remark 3.1.

THEOREM 3.4. Let $F: I \rightarrow \Omega^{n}$ be measurable and integrably bounded; then the following statements are equivalent:

(i ) $\mathscr{S}_{I}(F) \in \mathscr{C}^{n}(I)$.

(ii) $\mathscr{S}_{I}(F)$ is a nonvoid, weakly compact subset of $\mathscr{N} \mathscr{A} \mathscr{C}^{n}(I)$.

(iii) $F(t)$ is convex a.e. on $I$.

Proof. That (iii) implies both (i) and (ii) is an easy consequence of Theorem 3.1. For the remainder of the proof, consider the func- 
tion $\rho\left(F^{*}(\circ), F(\circ)\right)$. By virtue of Corollary 2.1, an argument similar to that of the first part of the proof of Theorem 2.3 permits the assertion that this function is measurable on $I$. Hence the set

$$
M \equiv\left\{t \in I \mid \bar{\rho}\left(F^{*}(t), F(t)\right)>0\right\}=\left\{t \in I \mid \rho\left(F^{*}(t), F(t)\right)>0\right\}
$$

is measurable. We need prove only that if $\mu(M)>0$ then $\mathscr{S}_{I}(F)$ is a proper subset of $\mathscr{S}_{I}\left(F^{*}\right)$. Indeed, in this event it follows from Corollary 3.5 that $\mathscr{S}_{I}(F) \notin \mathscr{H}^{n}(I)$ and, from Theorem 3.3, that $\mathscr{S}_{I}(F)$ is not weakly compact. Now we observe that minor modification of Hermes' proof [12] of Lemma 2.4 produces the following result: there exists a measurable function $w: I \rightarrow E^{n}$ satisfying $w(t) \in F^{*}(t)$ and $\alpha(w(t), F(t))=\rho\left(F^{*}(t), F(t)\right)$ for all $t \in I$. A function $w$ so determined thus satisfies $\alpha(w(t), F(t))>0$ on $M$. Hence, if $\mu(M)>0$ it follows that $\mathscr{F}_{I}(F)$ is a proper subset of $\mathscr{F}_{I}\left(F^{*}\right)$ and this in turn implies that $\mathscr{S}_{I}(F)$ is a proper subset of $\mathscr{S}_{I}\left(F^{*}\right)$ and the proof is complete.

\section{An existence theorem.}

THEOREM 4.1. Let $D$ be a nonvoid open subset of $E^{1} \times E^{n}$ and let $R: E^{1} \times E^{n} \rightarrow \Gamma^{n}$ satisfy conditions (i), (ii), (iii) of Lemma 2.8 on $D$; then for each $\left(t_{0}, x_{0}\right) \in D$ there exists a solution ${ }^{2}$ of

$$
\dot{x} \in R(t, x), x\left(t_{0}\right)=x_{0},
$$

and every solution of (2) may be continued to the boundary of $D$.

Proof. There is no loss of generality in assuming that $(0,0) \in D$ and proving the theorem in the case $\left(t_{0}, x_{0}\right)=(0,0)$. The proof is based on that of Hartman [10, Th. 2.1, p. 10]. Let $a, b>0$ be sufficiently small that $C \subset D$, where

$$
C=\left\{(t, x) \in E^{1} \times E^{n} \mid 0 \leqq t \leqq a ;\|x\| \leqq b\right\} .
$$

Define $\alpha=\max \left\{t \in[0, a] \mid \int_{0}^{t} h_{c}(\tau) d \tau \leqq b\right\} ; \quad$ evidently $\alpha \in(0, a]$. Let $\eta \in(0, \alpha]$ be fixed; then on $[0, \eta]$ the function whose value is $R(t, 0)$ is measurable and integrably bounded. By Theorem 3.1 there exists $w_{1} \in \mathscr{S}_{[0, \eta]}(R(\circ, 0))$ and we define a function $\chi_{\eta}$ on $[0, \eta]$ by

$$
\chi_{\eta}(t)=w_{1}(t), t \in[0, \eta] \text {. }
$$

There follows easily

$$
\left\|\chi_{\eta}(t)\right\| \leqq \int_{0}^{\eta} h_{c}(\tau) d \tau<b, t \in[0, \eta] ;
$$

2 I.e., an absolutely continuous function satisfying $\dot{x}(t) \in R(t, x(t))$ a.e. on an interval containing $t_{0}$ in its relative interior and satisfying $x\left(t_{0}\right)=x_{0}$. 


$$
\left\|\chi_{\eta}\left(t_{2}\right)-\chi_{\eta}\left(t_{1}\right)\right\| \leqq\left|\int_{t_{1}}^{t_{2}} h_{c}(\tau) d \tau\right|, t_{1}, t_{2} \in[0, \eta] .
$$

If $\eta<\alpha$, let $\eta^{1}=\min \{\alpha, 2 \eta\}$; then by Lemma 2.8 the function whose value is $R\left(t, \chi_{\eta}(t-\eta)\right)$ is measurable and integrably bounded on $\left[\eta, \eta^{1}\right]$. Hence by Theorem 3.1 there exists $w_{2} \in \mathscr{S}_{\left[\eta, \eta^{1]}\right.}\left(R\left(\circ, \chi_{\eta}(\circ-\eta)\right)\right)$. We extend $\chi_{\eta}$ to $\left[\eta, \eta^{1}\right]$ by defining

$$
\chi_{\eta}(t)=\chi_{\eta}(\eta)+w_{2}(t), t \in\left[\eta, \eta^{1}\right] ;
$$

it is easy to see that $\chi_{\eta}$ satisfies (4) on $\left[\eta, \eta^{1}\right]$, hence on $\left[0, \eta^{1}\right]$. If $\eta^{1}<\alpha$ the foregoing process may be iterated at most a finite number of steps to extend the definition of $\chi_{\eta}$ to $[0, \alpha]$ in such a way that the following property obtains:

$$
\begin{aligned}
\chi_{\eta} \in \mathscr{S}_{[0, \alpha]}\left(R^{\eta}(\circ)\right), & \text { where } R^{\eta}:[0, \alpha] \rightarrow \Gamma^{n} \text { is defined by } \\
R^{\eta}(t) & =R(t, 0), t \in[0, \eta], \\
R^{\eta}(t) & =R\left(t, \chi_{\eta}(t-\eta)\right), t \in(\eta, \alpha]
\end{aligned}
$$

with the family $\left\{R^{\eta} \mid \eta \in(0, \alpha]\right\}$ being uniformly integrably bounded and each member of the family measurable on $[0, \alpha]$.

Now let $\left\{\eta_{m}\right\}$ be a monotone null sequence of points in $[0, \alpha]$; then by property $\left({ }^{*}\right)$ and the Arzela-Ascoli theorem $\left\{\chi_{\eta_{m}}\right\}$ contains a subsequence (assume it is the original) which converges uniformly on $[0, \alpha]$ to a limit function, $\chi$, which is easily shown to be absolutely continuous (cf. the proof of Theorem 3.1). Equicontinuity of $\left\{\chi_{\eta_{m}}\right\}$ implies

$$
\lim \chi_{\eta_{m}}\left(t-\eta_{m}\right)=\chi(t), t \in[0, \alpha],
$$

so that by condition (i)

$$
\lim \rho\left(R^{\eta_{n}}(t), R(t, \chi(t))\right)=0, t \in[0, \alpha] .
$$

Thus from (*), (5) and Theorem 3.2 there follows

$$
\lim \sigma\left(\mathscr{S}_{[0, \alpha]}\left(R^{\eta_{m}}\right), \mathscr{S}_{[0, \alpha]}(R(\circ, \chi(\circ)))\right)=0 .
$$

Since $\chi_{\eta_{m}} \rightarrow \chi$ and $\mathscr{S}_{[0, \alpha]}(R(\circ, \chi(\circ)))$ is compact, $\left(^{*}\right)$ and (6) imply that

$$
\chi \in \mathscr{S}_{[0, \alpha]}(R(\circ, \chi(\circ))) \text {. }
$$

But (7) is equivalent to the assertion that $\chi(0)=0$ and, a.e. on $[0, \alpha]$,

$$
\dot{\chi}(t) \in R(t, \chi(t))
$$

and the proof of existence is complete. The continuability assertion follows in a straightforward way from [2, Th. 4]. 
(i), (ii), (iii) of Lemma 2.8 are replaced by (iv) $R$ is continuous on $D$, then the conclusion of that theorem remains valid.

Proof. That (iv) implies (i) is obvious; that (iv) implies (ii) is a consequence of Lemma 2.3. Finally, (iii) follows from (iv) by setting

$$
h_{c}(t)=\max \{\max \{\|\xi\| \mid \xi \in R(\tau, x)\} \mid(\tau, x) \in C\}, t \in E^{1} .
$$

REMARK 4.1. The demonstration that all solutions of (2) may be continued over the interval $[0, \alpha]$, defined in the proof of Theorem 4.1, is exactly like the corresponding proof for ordinary differential equations. The compactness of the solution family as a subset of $\mathscr{C}^{n}([0, \alpha])$ is then an easy consequence of Theorem 3.2 ; this again is a parallel to the corresponding argument for ordinary differential equations. Invoking [5, Th. 1] and Corollary 2.1, only slight modification of the proof of Theorem 4.1 is needed to establish the more general Pliś-Castaing existence theorem [17], [5].

5. The Huygens derivative.

DEFINITION 5.1. Let $\mathscr{S} \in \mathscr{H}^{n}(I)$; given $t \in I$, if there exists $S(t) \in \Gamma^{n}$ such that

$$
\lim _{\eta \rightarrow 0+} \eta^{-1} \rho(G(t+\eta ; \mathscr{S}), G(t ; \mathscr{S})+\eta S(t))=0
$$

then $S(t)$ is called a right hand (Huygens) derivative of $\mathscr{S}$ at $t$. If there exists $V(t) \in \Gamma^{n}$ such that

$$
\lim _{\eta \rightarrow 0+} \eta^{-1} \rho(G(t-\eta ; \mathscr{S})+\eta V(t), G(t ; \mathscr{S}))=0
$$

the $V(t)$ is called a left hand (Huygens) derivative of $\mathscr{S}$ at $t$.

LEMma 5.1. The one-sided Huygens derivatives of $\mathscr{S} \in \mathscr{H}^{n}(I)$ are unique.

Proof. We give the proof for right hand derivatives, the proof for left hand derivatives being similar. Let $R(t), S(t)$ be right hand derivatives of $\mathscr{S}$ at $t$; then for $\eta>0$ it follows from Lemma 1.3 and the triangle law that

$$
\begin{aligned}
\rho(R(t), S(t))= & \eta^{-1} \rho(\eta R(t), \eta S(t))=\eta^{-1} \rho(G(t ; \mathscr{S})+\eta R(t), G(t ; \mathscr{S})+\eta S(t)) \\
\leqq & \eta^{-1} \rho(G(t+\eta ; \mathscr{S}), G(t ; \mathscr{S})+\eta R(t)) \\
& +\eta^{-1} \rho(G(t+\eta ; \mathscr{S}), G(t ; \mathscr{S})+\eta S(t)) .
\end{aligned}
$$

By hypothesis, the limit, as $\eta \rightarrow 0+$, of the rightmost member is zero so that $\rho(R(t), S(t))=0$. 
Definition 5.2. When these exist, the right hand and left hand derivatives at $t$ of $\mathscr{S} \in H^{n}(I)$ will be denoted by $\left(D^{+} \mathscr{S}\right)(t)$ and $\left(D^{-} \mathscr{S}\right)(t)$ respectively. If the one-sided derivatives of $\mathscr{S}$ at $t$ both exist and are equal, their common value is called the Huygens derivative of $\mathscr{S}$ at $t$ and is denoted by $(D \mathscr{S})(t)$.

Lemma 5.2. If $F: I \rightarrow \Gamma^{n}$ is measurable and integrably bounded then

$$
\nu\left(G\left(t ; \mathscr{S}_{I}(F)\right), p\right)=\int_{0}^{t} \nu(F(\tau), p) d \tau, t \in I, p \in E^{n} .
$$

Proof. Let us condense notation by defining

$$
\begin{aligned}
& \omega(t, p)=\nu\left(G\left(t ; \mathscr{S}_{I}(F)\right), p\right), \\
& \lambda(t, p)=\nu(F(t), p) .
\end{aligned}
$$

Then the assertion of the lemma is that $\omega(t, p)=\int_{0}^{t} \lambda(\tau, p) d \tau, t \in I$, $p \in E^{n}$. By an argument similar to that for Theorem 3.1 it follows that $\lambda(\circ, p)$ is summable for each $p \in E^{n}$ so that $\int_{0}^{t} \lambda(\tau, p) d \tau$ is well defined. If $\sigma \in G\left(t ; \mathscr{S}_{I}(F)\right)$ then there exists $u^{*} \in \mathscr{F}_{I}(F)$ such that $\sigma=\int_{0}^{t} u^{*}(\tau) d \tau$; hence

$$
\sigma \circ p=\int_{0}^{t} u^{*}(\tau) \circ p d \tau \leqq \int_{0}^{t} \lambda(\tau, p) d \tau, t \in I, p \in E^{n}
$$

We infer that $\omega(t, p) \leqq \int_{0}^{t} \lambda(\tau, p) d \tau$. For the proof of the reverse inequality let $h$ be the integrable bound on $F$; then for $\eta>0$ and $\|\mathrm{p}\|=1,(h(t)+\eta) \notin F(t)$ on $I$. For suppose the contrary; then

$$
h(t)<h(t)+\eta=\|(h(t)+\eta) p\| \leqq h(t),
$$

which is absurd. Let $q(t, \eta, p)$ be the unique point in the boundary of $F(t)$ nearest $(h(t)+\eta) p$; then by virtue of Lemma 2.5, $q(\circ, \eta, p)$ is summable and

$$
\int_{0}^{t} \lambda(\tau, p) d \tau=\int_{0}^{t} q(\tau, \eta, p) \circ p d \tau=\left(\int_{0}^{t} q(\tau, \eta, p) d \tau\right) \circ p \leqq \omega(t, p) .
$$

This completes the proof.

THEOREM 5.1. If $F: I \rightarrow \Omega^{n}$ is measurable and integrably bounded then a.e. on $I,\left(D \overline{\mathscr{S}_{I}(\bar{F})}\right)(t)=F^{*}(t)$.

Proof. By virtue of Corollary 3.4, $\left(D \overline{\mathscr{S}_{I}\left(F^{\prime}\right)}\right)(t)$ exists if and only if $\left(D \mathscr{S}_{I}\left(F^{*}\right)\right)(t)$ exists; moreover, the two have the same value. It is thus sufficient to show that $\left(D \mathscr{S}_{1}\left(F^{*}\right)\right)(t)=F^{*}(t)$ a.e. on $I$; we shall 
carry out the proof for $D^{+}$, the proof for $D^{-}$being similar. For $\eta>0$ we find that with $\omega, \lambda$ being as defined in the proof of Lemma 5.2 ,

$$
\begin{aligned}
& \eta^{-1} \rho\left(G\left(t+\eta ; \mathscr{S}_{I}\left(F^{*}\right)\right), G\left(t ; \mathscr{S}_{I}\left(F^{*}\right)\right)+\eta F^{*}(t)\right) \\
= & \eta^{-1} \max \{\mid \omega(t+\eta, p)-[\omega(t, p)+\eta \lambda(t, p)]\|\| p \|=1\} \\
= & \left.\eta^{-1} \max \left\{\mid \int_{t}^{t+\eta} \lambda(\tau, p) d \tau-\eta \lambda t, p\right)||\|p\|=1\right\} \quad \text { (by Lemma 5.2) } \\
= & \eta^{-1} \max \left\{\left|\int_{t}^{t+\eta}[\lambda(\tau, p)-\lambda(t, p)] d \tau\right| \mid\|p\|=1\right\} \\
\leqq & \eta^{-1} \int_{t}^{t+\eta} \Delta\left(F^{*}(\tau), F^{*}(t)\right) d \tau \leqq \eta^{-1} \int_{t}^{t+\eta} \rho(F(\tau), F(t)) d \tau
\end{aligned}
$$

(by Lemma 1.3 (ii)).

The proof is completed by invoking Theorem 2.3.

CoROLlary 5.1. If $F_{i}: I \rightarrow \Omega^{n}, i=1,2$, are measurable and integrably bounded, a necessary and sufficient condition that the closures of $\mathscr{S}_{I}\left(F_{1}\right)$ and $\mathscr{S}_{1}\left(F_{2}\right)$ be equal is that $F_{1}^{*}(t)=F_{2}^{*}(t)$ a.e. on $I$.

Proof. (Sufficiency.) Evidently $\mathscr{S}_{1}\left(F_{1}^{*}\right)=\mathscr{S}_{1}\left(F_{2}^{*}\right)$ and the assertion follows from Corollary 3.4.

(Necessity.) By hypothesis, Corollary 3.4 and Theorem 5.1, a.e. on $I$ we have

$$
\left.\left.F_{1}^{*}(t)=\left(D \overline{\mathscr{S}_{I}\left(F_{1}\right.}\right)\right)(t)=\left(D \overline{\mathscr{S}_{I}\left(F_{2}\right.}\right)\right)(t)=F_{2}^{*}(t) .
$$

For $t_{1}, t_{2} \in I$, let us set

$$
\int_{t_{1}}^{t_{2}} F(\tau) d \tau=\left\{\int_{t_{1}}^{t_{2}} q(\tau) d \tau \mid q \in \mathscr{F}_{I}(F)\right\}
$$

where $F: I \rightarrow \Omega^{n}$. It is not difficult to verify that for $\eta>0$

$$
G\left(t+\eta ; \mathscr{S}_{I}(F)\right)=G\left(t ; \mathscr{S}_{I}(F)\right)+\int_{\tau}^{t+\eta} F(\tau) d \tau, t, t+\eta \in I,
$$

and

$$
G\left(t-\eta ; \mathscr{S}_{I}(F)\right)+\int_{t-\eta}^{t} F(\tau) d \tau=G\left(t ; \mathscr{S}_{I}(F)\right), t, t-\eta \in I .
$$

Thus if $F: I \rightarrow \Omega^{n}$ is measurable and integrably bounded there follow from Lemma 3.3, Lemma 1.3 and the foregoing identities, both

$$
\eta^{-1} \rho\left(G\left(t+\eta ; \mathscr{S}_{I}(F)\right), G\left(t ; \mathscr{S}_{I}(F)\right)+\eta F^{*}(t)\right)=\rho\left(\eta^{-1} \int_{t}^{t+\eta} F(\tau) d \tau, F^{*}(t)\right)
$$




$$
\eta^{-1} \rho\left(G\left(t-\eta ; \mathscr{S}_{I}(F)\right)+\eta F^{*}(t), G\left(t ; \mathscr{S}_{I}(F)\right)\right)=\rho\left(\eta^{-1} \int_{t-\eta}^{t} F(\tau) d \tau, F^{*}(t)\right)
$$

when $\eta>0$. Together with Theorem 5.1, these last formulae establish the following generalization of [11, Lemmas 1.2, 1.3].

COROLlaRY 5.2. If $F: I \rightarrow \Omega^{n}$ is measurable and integrably bounded then, a.e. on $I$,

$$
\lim _{\eta \rightarrow 0} \rho\left(\eta^{-1} \int_{t}^{t+\eta} F(\tau) d \tau, F^{*}(t)\right)=0
$$

REMARK 5.1. Note that now Corollary 5.1 appears as a generalization of [11, Th. 1.1].

\section{REFERENCES}

1. R. J. Aumann, Integrals of set-valued functions, J. Math. Anal. Appl. 12 (1965), $1-12$.

2. J. Bebernes, W. Fulks, G. H. Meisters, Differentiable paths and continuation of solutions of differential equations, J. Diff. Equations 2 (1966), 102-106.

3. T. F. Bridgland, Jr., On the problem of approximate synthesis of optimal controls, J. SIAM Control 5 (1967), 326-344.

4. Contributions to the theory of generalized differential equations, Math. Systems Theory, 3 (1969), 17-50.

5. C. Castaing, Sur les equations differentielles multivoques, C. R. Acad. Sci. Paris (A) 263 (1966), 63-66.

6. N. Dunford and J. T. Schwartz, Linear operators, part I: general theory, Interscience, New York, 1958.

7. H. G. Eggleston Convexity, Cambridge Univ. Press, Cambridge, 1958.

8. A. F. Filippov, On certain questions in the theory of optimal control, J. SIAM Control (A) 1 (1962), 76-84.

9. - Differential equations with many-valued discontinuous right-hand side,

Soviet Math. 4 (1963), 941-945.

10. P. Hartman, Ordinary differential equations, J. Wiley \& Sons, New York, 1964.

11. H. Hermes, Calculus of set valued functions and control, J. Math. Mech. 18 (1968), 47-60.

12. appear)

13. H. Kudo, Dependent experiments and sufficient statistics, Nat. Sci. Rept. Ochanomizu Univ., Tokyo 4 (1954), 151-163.

14. C. Kuratowski, Topologie I, Monografie Mat. Tom XX, Warsaw, 1948.

15. I. P. Natanson, Theory of functions of a real variable, Vol. I, tr. L. F. Boron, F. Ungar Publishing Co., New York, 1961.

16. A. Pliś, Remark on measurable set-valued functions, Bull. Acad. Polon. Sci., Ser. Sci., Math., Astr., Phys. 9 (1961), 857-859.

17. —, Measurable orientor fields, Bull. Acad. Polon. Sci. Ser. Sci., Math., Astr., Phys. 13 (1965), 565-569. 
18. H. Richter, Verallgemeinerung eines in der Statistik benotigen Satzes der Masstheorie, Math. Ann. 150 (1963), 85-90; 440-441.

19. F. A. Valentine, Convex sets, McGraw-Hill, New York, 1964.

Received January 3, 1969, and in revised form September 11, 1969. This research was sponsored by the National Science Foundation under grant GP-8921.

DREXEL UNIVERSITY 


\section{PACIFIC JOURNAL OF MATHEMATICS}

\section{EDITORS}

H. SAMELSON

Stanford University

Stanford, California 94305

\section{RichaRd PIERCe}

University of Washington

Seattle, Washington 98105
J. DUGUNDJI

Department of Mathematics

University of Southern California

Los Angeles, California 90007

RICHARD ARENS

University of California

Los Angeles, California 90024

\section{ASSOCIATE EDITORS}

E. F. BECKENBACH

B. H. NeUManN

F. WOLF

K. YosHIDA

\section{SUPPORTING INSTITUTIONS}

UNIVERSITY OF BRITISH COLUMBIA CALIFORNIA INSTITUTE OF TECHNOLOGY UNIVERSITY OF CALIFORNIA MONTANA STATE UNIVERSITY

UNIVERSITY OF NEVADA

NEW MEXICO STATE UNIVERSITY

OREGON STATE UNIVERSITY

UNIVERSITY OF OREGON

OSAKA UNIVERSITY

UNIVERSITY OF SOUTHERN CALIFORNIA
STANFORD UNIVERSITY

UNIVERSITY OF TOKYO

UNIVERSITY OF UTAH

WASHINGTON STATE UNIVERSITY

UNIVERSITY OF WASHINGTON

${ }^{*} \quad{ }^{*} \quad{ }^{*}$
AMERICAN MATHEMATICAL SOCIETY
CHEVRON RESEARCH CORPORATION
TRW SYSTEMS
NAVAL WEAPONS CENTER




\section{Pacific Journal of Mathematics}

\section{Vol. 33, No. $1 \quad$ March, 1970}

Mir Maswood Ali, On some extremal simplexes ................... 1

Silvio Aurora, On normed rings with monotone multiplication........... 15

Silvio Aurora, Normed fields which extend normed rings of integers....... 21

John Kelly Beem, Indefinite Minkowski spaces..................... 29

T. F. Bridgland, Trajectory integrals of set valued functions ........... 43

Robert Jay Buck, A generalized Hausdorff dimension for functions and sets ......................................... 69

Vlastimil B. Dlab, A characterization of perfect rings . . . . . . . . . . . . 79

Edward Richard Fadell, Some examples in fixed point theory ............ 89

Michael Benton Freeman, Tangential Cauchy-Riemann equations and uniform approximation ............................. 101

Barry J. Gardner, Torsion classes and pure subgroups ................ 109

Vinod B. Goyal, Bounds for the solution of a certain class of nonlinear

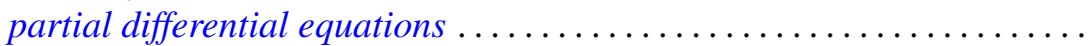

Fu Cheng Hsiang, On C, 1 summability factors of Fourier series at a given

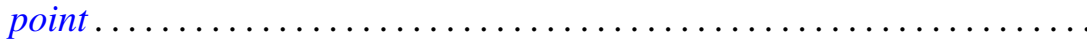

Lawrence Stanislaus Husch, Jr., Homotopy groups of PL-embedding

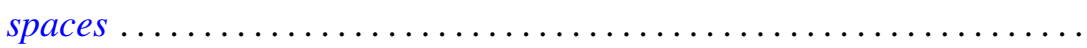

Daniel Ralph Lewis, Integration with respect to vector measures..........

Marion-Josephine Lim, $\mathscr{L}-2$ subspaces of Grassmann product spaces

Stephen J. Pierce, Orthogonal groups of positive definite multilinear functionals

W. J. Pugh and S. M. Shah, On the growth of entire functions of bounded index.

Siddani Bhaskara Rao and Ayyagari Ramachandra Rao, Existence of triconnected graphs with prescribed degrees . . .

Ralph Tyrrell Rockafellar, On the maximal monotonicity of subdifferential

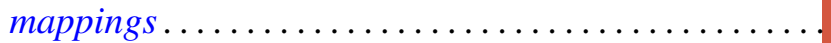

R. Shantaram, Convergence of a sequence of transformations of distribution functions. II ...............................

Julianne Souchek, Rings of analytic functions..............

Ted Joe Suffridge, The principle of subordination applied to functions of several variables...

Wei-lung Ting, On secondary characteristic classes in cobordism

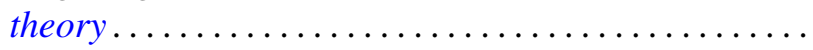

Pak-Ken Wong, Continuous complementors on $B^{*}$-algebras ...

Miyuki Yamada, On a regular semigroup in which the idempotents form a band. 